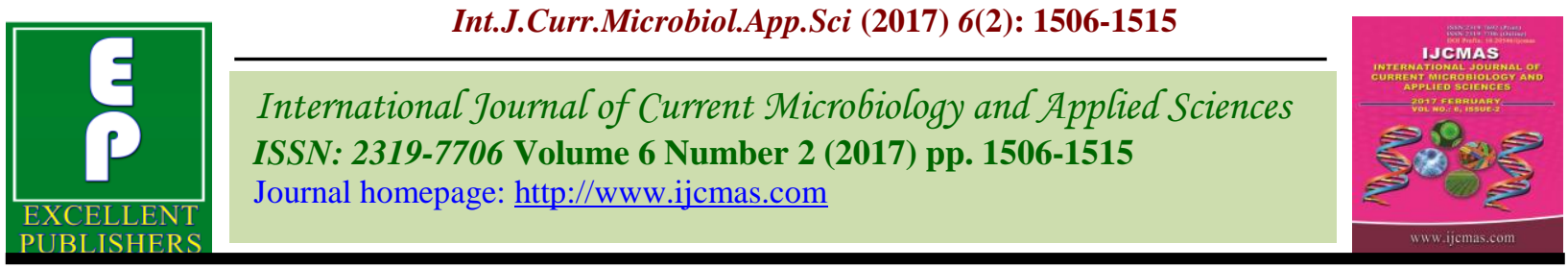

Original Research Article

http://dx.doi.org/10.20546/ijcmas.2017.602.168

\title{
Stability Analysis and Genotype X Environment Interaction of Quality Traits in Tomato (Solanum lycopersicum L.)
}

\author{
Sonam Spaldon*, R.K. Samnotra, Rinchan Dolkar and Deshraj Choudhary \\ Division of Vegetable Science and Floriculture, Sher-e-Kashmir University of Agricultural \\ Sciences and Technology of Jammu, Main Campus, Chatha, Jammu 180009, India \\ *Corresponding author
}

\section{A B S T R A C T}

Keywords

Stability, Quality traits, Solanum Lycopersicum L., adaptability.

Article Info

Accepted:

24 January 2017

Available Online:

10 February 2017
The present investigation was conducted during spring summer season of 20122013 and 2013-2014 over six environments to study stability of 25 genotype of tomato for quality traits. Genotypes $\times$ environment interaction was significant for lycopene, betacarotene and ascorbic acid which indicated the differential response of genotype to various environment conditions and non significant for number of locules per fruit, pericarp thickness, total soluble solids and fruit $\mathrm{pH}$. None of the genotypes was stable for all the traits and stability for one trait was independent of stability for other traits. Genotype Rupali was found stable for number of locules per plant, while genotypes Karan for pericarp thickness. The genotypes Kubergeeta, Arka Saurabh and Arka Meghali were identified as stable genotypes for fruit $\mathrm{pH}$. The genotypes Aditya, Karan, Arka Vikas and Arka Meghali can be utilized in further quality breeding program.

\section{Introduction}

Tomato (Solanum lycopersicum L.) is an important warm season, self pollinated vegetable crop grown both for fresh and food processing market (Nwosu et al., 2014). It belongs to the family of Solanaceae (Das et al., 2011) and native of Peru Equador region (Rick, 1969). It is a rich source of vitamins A, $\mathrm{B}$ and $\mathrm{C}$ and minerals (Akinfasoy et al., 2011). Tomato is processed into various value addition products like sauce, ketchup, juice, soup, puree, margarine, paste and whole canned tomatoes (Maitidevi and Kathmandu, 2008). Consumption of tomatoes and its products has been linked to reduce carcinogenesis, particularly prostate and mouth cancer due to the presence of antioxidants, beta carotene and lycopene contents (Giovannucci, 2002; Miller et al., 2002; Bai and Lindhot, 2007). Ascorbic acid may play a key role in delaying the pathogenesis of a variety of degenerative diseases, such as cardiovascular disease, certain cancers, cataracts and it also prevents DNA mutation induced by oxidative stress (Marchioli et al., 2001; Lutsenko et al., 2002). Phenotypically stable genotypes are of great importance, because the environmental condition varies from year to year/ region to region. Wide adaptation to particular environment and consistent performance of genotypes is one of the main objectives in breeding programme. Although number of 
genotypes has been recommended for cultivation, yet the information on their stability for quality traits is lacking for varied agro climatic of subtropical conditions of Jammu. The quality of the tomato genotypes plays an important role in deciding the suitability of the genotype for long distance transportation, processing purpose and for fresh market. Considering these requirements, quality parameters, viz., number of locules fruit $^{-1}$, Pericarp thickness $(\mathrm{mm})$, Total soluble solids $\left({ }^{0} \mathrm{~B}\right)$, Lycopene $(\mathrm{mg} / 100 \mathrm{~g})$, Beta carotene (mg/100g), Ascorbic acid (mg/100g) and $\mathrm{pH}$ were estimated in present investigation.

\section{Materials and Methods}

Twenty five genotypes of tomato were tested under six environment comprised of three dates of transplanting for two years during spring summer season of 2012-2013 and 2013-2014 at the experimental farm of Division of Vegetable Science and Floriculture, SKUAST-Jammu which is situated at $32^{\circ} 40^{\prime} \mathrm{N}$ latitude and $74^{\circ} 58^{\prime} \mathrm{E}$ longitude and has an elevation of $332 \mathrm{~m}$ above mean sea level. The place experiences hot dry summer, hot and humid rainy season and cold winter months, the maximum temperature goes up to $45^{\circ} \mathrm{C}$ during summers (May to June) and minimum temperature falls to $1^{\circ} \mathrm{C}$ during winters. The information on climatic conditions during the crop season was recorded in Division of Agrometerology, Chatha. The individual experiment was conducted in Randomized block design with three replications. The uniform, healthy seedlings were planted on ridges maintaining inter and intra row spacing of 60 and $45 \mathrm{~cm}$ respectively. Genotypes were assessed for their stability of performance over environments in accordance with method described by Eberhart and Russel (1966). In this model a stable genotype is being one with a regression slope near to one, deviation from regression line being close to zero and higher mean yield. Data were analyzed by window stat software.

Quality parameters, viz., number of locules fruit $^{-1}$, Pericarp thickness $(\mathrm{mm})$, Total soluble solids $\left({ }^{0} \mathrm{~B}\right)$, Lycopene $(\mathrm{mg} / 100 \mathrm{~g})$, Beta carotene $(\mathrm{mg} / 100 \mathrm{~g})$, Ascorbic acid $(\mathrm{mg} / 100 \mathrm{~g})$ and $\mathrm{pH}$ were estimated for all the genotypes. Number of locules was counted from five fruits taken at random and cut transversely in the middle and were sliced at the equatorial plane to measure pericarp thickness with the help of vernier caliper. A drop of tomato juice from each reference was put on the prism of hand refractometer and reading on per cent scale was noted for total soluble solids estimation. Beta carotene content (mg/100g) was estimated through the procedure given by (Sadasivam and Manickam, 1992); Lycopene content (mg/100g) and Ascorbic acid content (mg/100g) by Rangana (1976).

\section{Results and Discussion}

Analysis of variance over environments (Table 1) revealed that mean sum of squares due to genotypes were highly significant for all the traits except for fruit $\mathrm{pH}$ under studied which indicated the presence of genetic variability among the genotypes involved in the study for all the traits studied. This provides an ample opportunity for selecting suitable genotypes with high mean for all the traits of interest. These results are in accordance with those of Jyothi et al., (2012); Mane et al., (2010) and Aravindakumar et al., (2001). The mean square due to environments were significant for all the traits namely pericarp thickness, total soluble solids, betacarotene, ascorbic acid and $\mathrm{pH}$ which indicated the presence of variable environments in expression of all the traits. The presence of genotypes $\times$ environment interaction was also significant for lycopene, betacarotene and ascorbic acid which 
indicated the differential response of genotype to various environment conditions. Whereas it was found no significant for number of locules per fruit, pericarp thickness, total soluble solids and fruit $\mathrm{pH}$. This results is in conformity with the findings of Jyothi et al., (2012)

The pooled analysis of variance for various traits in tomato over six environments (Table 2) revealed that the variation due to $G \times E$ interaction has been partitioned into two, the predictable component due to linear regression and the unpredictable one due to pooled deviations from regression. Mean sum of squares due to Environment + (Genotype $\times$ Environment) was observed significant for number of locules per fruit, total soluble solids, betacarotene, ascorbic acid and $\mathrm{pH}$ which depicted the existence of genotype $x$ environment interaction. The linear contribution of environment on the performance of genotypes was significant for almost all the traits under studied except for lycopene indicating that environmental effects were predictable. This result agrees with the findings of Jyothi et al., (2012).

The mean square due to genotype $x$ environment (Linear) when tested against pooled deviation were significant for almost all the traits viz., number of locules per fruit, pericarp thickness, total soluble solids, betacarotene, ascorbic acids and $\mathrm{pH}$. This indicated significant rate of linear response of the genotypes to environmental changes for these traits. These results were in conformity with earlier reports of Jyothi et al.,(2012) for total soluble solids and $\mathrm{pH}$; Prasanna et al., (2007) for number of locules and total soluble solid; Non significant effect of genotype $x$ environment (linear) for lycopene indicated that the different genotypes did not differ genetically in their response to different environments. The pooled deviation when tested against pooled error was found significant for all the traits under studied which indicated the important contribution of non predictable component. Similar results were recorded in earlier reports of Mane et $a l$. , (2010) for pericarp thickness and number of locules.

Three genotypes Lehar, Heem Sohna, and Leh Local exhibited regression coefficient lesser than unity $\left(b_{i}<1\right)$ with non significant deviation from regression line so are suitable under unfavourable environment. Genotype Kubergeeta which are comparable to average mean showed regression coefficient greater than one $\left(b_{i}>1\right)$ with non significant deviation from regression line reflecting its preference under favourable environments. Amongst all the genotypes, genotype Rupali showed regression coefficient close to one with non significant deviation from regression line indicates its adaptation to all the environments. The findings are in corroborate with Thapliyal and Singh (2009) in three genotypes; Mane et al., (2010) reported four genotypes as stable this trait under study. Whereas genotype Pusa Ruby which recorded regression coefficient close to unity with significant deviation from regression line. Varied response of genotypes due to change in environments for number of locules in the present investigation is in contradiction with findings of Aravindakumar et al.,(2001) who revealed only two genotypes were found stable and rest of 6 genotypes were suitable to poor environments (Tables 3 and 4).

Pericarp thickness is an important feature of the tomato fruit, as genotypes with thicker pericarp are better to withstand long distance transportation and remain firm for a longer period, when compared to thinly fleshed tomatoes (Kumari and Sharma, 2011). Genotypes Karan had regression coefficient close to one with non significant deviation from regression so are widely adapted to all the environments. These results are in 
accordance with Kalloo et al., (1998) in four genotypes; Prasanna et al., (2007) reported stable performance for this trait in seven genotypes under study; Maharishi had regression coefficient greater than unity with non significant deviation from regression line which indicates its adaptation to favourable environment. These results are in conformity with Mane et al., (2010) in eight genotypes; Aravindakumar et al.,(2001) in which he revealed three genotypes as stable performance and two genotypes under favourable condition. Genotype Tokita are comparable to average mean with regression coefficient less than unity which are suitable to unfavourable environments.

Total soluble solids content of tomato fruits is essential for processing purpose. $50 \%$ to $65 \%$ of soluble solids contents are sugars, glucose and fructose and their amount and proportion influences the organoleptic quality of tomatoes (Adedeji et al., 2006). High total soluble solids are desirable to higher yield of processed products. Genotype Arka Vikas recorded the highest total soluble solid (5.02 ${ }^{0} \mathrm{~B}$ ) and lowest was recorded by Tokita (3.36 $\left.{ }^{0} \mathrm{~B}\right)$. Among hybrids it ranged from 4.39 to 3.36 ${ }^{0} \mathrm{~B}$ and 5.02- $4.03{ }^{0} \mathrm{~B}$ in open pollinated genotypes. These findings are in support to the findings of Dufera (2013) where TSS range from $1.68-4.92{ }^{0} \mathrm{~B}$ in 21 genotypes; Jyothi et al., (2012) found TSS range from $4.30-5.67^{0} \mathrm{~B}$ in 12 genotypes under study; Hazarika and Phookan (2009) obtained range from 4.24-6.54 ${ }^{0} \mathrm{~B}$; Revanasiddappa (2008) observed TSS of 4.40 brix and Cantore et al., (2008)observed highest soluble solids content $\left(5^{0}\right.$ Brix). Genotype Marglobe and Solan Lalima had regression coefficient close to one showing average in response and were unstable due to significant deviation from regression line. None of the genotypes were found stable for this trait as all the genotypes showed significant deviation from regression line. Genotype Pusa Ruby recorded maximum
TSS having significant regression coefficient lesser than one with significant deviation from regression line. Similar findings have been reported by Aravindakumar et al., (2001) in genotype Pusa Ruby.

An antioxidant present in tomato fruit i.e. lycopene is essential for colour of fruits. The higher lycopene content genotypes are preferred for fresh market. The genotypes with the highest contents of lycopene and highest antioxidant activity represents a valuable genotype not only for improving the status of dietary antioxidants in our diet but also for increasing nutritional value through germplasm enhancement programmes (George et al., 2004). In the present study, genotype Aditya had maximum lycopene $(5.22 \mathrm{mg} / 100 \mathrm{~g})$ content as compared to the average mean $(2.94 \mathrm{mg} / 100 \mathrm{gm}$ and minimum was noticed in Swarna Lalima (1.36mg/100g). Among hybrid it ranges from 5.22 to $1.79 \mathrm{mg} / 100 \mathrm{~g}$ as compared to open pollinated genotypes which ranges from 3.89 to 1.36 $\mathrm{mg} / 100 \mathrm{~g}$. These findings are in agreement with the results of Cheema et al., (2013) who recorded maximum lycopene content in hybrids varying from $5.25 \mathrm{mg} / 100 \mathrm{~g}$ $2.75 \mathrm{mg} / 100 \mathrm{~g}$ under open field conditions; Dufera (2013) observed in the range of 0.903.29 in 21 genotypes under study; Panthee et al., (2012) obtained in the range of 5.80-21.80 in 42 diverse tomato genotypes; Jyothi et al.,(2012) recorded lycopene content varying from $5.64 \mathrm{mg} / 100 \mathrm{~g}$ to $3.07 \mathrm{mg} / 100 \mathrm{~g}$ in 12 genotypes.

Only one genotype viz., Marglobe which is comparable with average mean displayed regression coefficient less than one with non significant regression coefficient line which reflects its adaptability to unfavourable environments. Similar results had also been obtained by Jyothi et al., (2012) in genotype PTR-1. 
Table.1 Analysis of variance over environments for various quality traits in tomato (Solanum lycopersicum L.)

\begin{tabular}{|c|c|c|c|c|c|c|c|c|}
\hline $\begin{array}{c}\text { Source of } \\
\text { variation }\end{array}$ & Df & $\begin{array}{c}\text { Number } \\
\text { of } \\
\text { locules } \\
\text { fruit }^{-1}\end{array}$ & $\begin{array}{c}\text { Pericarp } \\
\text { thickness } \\
(\mathbf{m m})\end{array}$ & $\begin{array}{c}\text { Total soluble } \\
\left.\text { solids } \mathbf{(}^{\mathbf{0}} \mathbf{B}\right)\end{array}$ & $\begin{array}{c}\text { Lycopene } \\
(\mathbf{m g} / \mathbf{1 0 0 g})\end{array}$ & $\begin{array}{c}\text { Beta } \\
\text { carotene } \\
(\mathbf{m g} / \mathbf{1 0 0 g})\end{array}$ & $\begin{array}{c}\text { Ascorbic } \\
\text { acid } \\
(\mathbf{m g} / \mathbf{1 0 0 g})\end{array}$ & $\mathbf{p H}$ \\
\hline Genotype & 24 & $7.60^{* *}$ & $15.65^{* *}$ & $2.32^{*}$ & $12.39^{* *}$ & $54.50^{* *}$ & $380.93^{* *}$ & 0.30 \\
\hline Environment & 5 & 2.01 & $3.91^{*}$ & $4.46^{*}$ & 1.080 & $17.39^{* *}$ & $44.71^{* *}$ & 0.45 \\
\hline $\begin{array}{c}\text { Genotype } \times \\
\text { Environment }\end{array}$ & 120 & 0.83 & 1.77 & 0.85 & $3.45^{* *}$ & $14.60^{* *}$ & $15.69^{* *}$ & 0.01 \\
\hline Error & 300 & 0.05 & 0.05 & 0.017 & 0.02 & 0.13 & 0.265 & 0.00 \\
\hline S.E & 0.13 & 0.19 & 0.10 & 0.11 & 0.30 & 0.42 & 0.04 \\
\hline CD at 5\% & 0.25 & 0.38 & 0.21 & 0.23 & 0.60 & 0.82 & 0.09 \\
\hline CD at 1\% & 0.33 & 0.50 & 0.27 & 0.30 & 0.79 & 1.08 & 0.12 \\
\hline
\end{tabular}

*significant at $5 \%$ level of significance, $* *$ significant at $1 \%$ level of significance

Table.2 Mean Squares due to different source of variation for various quality traits in tomato (Solanum lycopersicum L.)

\begin{tabular}{|c|c|c|c|c|c|c|c|c|}
\hline Source of variation & Df & $\begin{array}{l}\text { Number } \\
\text { of locules } \\
\text { fruit }^{-1}\end{array}$ & $\begin{array}{l}\text { Pericarp } \\
\text { thickness } \\
(\mathrm{mm})\end{array}$ & $\begin{array}{l}\text { Total } \\
\text { soluble } \\
\text { solids }\left({ }^{0} \mathbf{B}\right)\end{array}$ & $\begin{array}{l}\text { Lycopene } \\
\text { (mg/100g) }\end{array}$ & $\begin{array}{l}\text { Beta } \\
\text { carotene } \\
(\mathrm{mg} / 100 \mathrm{~g})\end{array}$ & $\begin{array}{l}\text { Ascorbic } \\
\text { acid } \\
\text { (mg/100g) }\end{array}$ & pH \\
\hline $\begin{array}{l}\text { Replication within } \\
\text { Environment }\end{array}$ & 12 & 0.03 & 0.05 & 0.00 & 0.01 & 0.07 & 0.19 & 0.00 \\
\hline Genotypes & 24 & $2.53 * *$ & $4.91 * *$ & $0.77 * *$ & $4.13 * *$ & $18.17 * *$ & $127.56^{* *}$ & $0.10 * *$ \\
\hline $\begin{array}{l}\text { Environment }+(\text { Genotype } \\
\times \text { Environment })\end{array}$ & 125 & $0.29 * *$ & 0.63 & $0.33 *$ & 1.11 & $4.90 *$ & $5.72 *$ & $0.01 * *$ \\
\hline Environments & 5 & $0.67 * *$ & 1.12 & $1.48 * *$ & 0.35 & 5.78 & $15.59 * *$ & $0.15^{* *}$ \\
\hline Genotype $\times$ Environment & 120 & $0.27 * *$ & 0.61 & 0.28 & 1.14 & $4.87 *$ & $5.31 *$ & $0.00 * *$ \\
\hline Environments (Linear) & 1 & $3.34 * *$ & $5.61 * *$ & $7.41 * *$ & 1.78 & $28.90 * *$ & $77.98 * *$ & $0.74 * *$ \\
\hline $\begin{array}{l}\text { Genotype } \times \text { Environment } \\
(\text { Linear })\end{array}$ & 24 & $0.67 * *$ & $1.03 * *$ & $0.49 * *$ & 1.23 & $10.23^{* *}$ & $10.73 * *$ & $0.01 * *$ \\
\hline Pooled deviation & 100 & $0.17 * *$ & $0.48 * *$ & $0.22 * *$ & $1.08 * *$ & $3.38 * *$ & $3.80 * *$ & $0.003^{* *}$ \\
\hline Pooled error & 288 & 0.01 & 0.02 & 0.006 & 0.007 & 0.04 & 0.14 & 0.001 \\
\hline
\end{tabular}

*significant at $5 \%$ level of significance, $* *$ significant at $1 \%$ level of significance 
Table.3 Mean value, regression coefficient (bi) and variation due to deviation (s2di) for 25 tomato genotypes

\begin{tabular}{|c|c|c|c|c|c|c|c|c|c|c|c|c|c|}
\hline \multirow[b]{2}{*}{$\begin{array}{l}\text { S. } \\
\text { No. }\end{array}$} & \multirow[b]{2}{*}{ Genotype } & \multicolumn{3}{|c|}{ Number of locules fruit ${ }^{-1}$} & \multicolumn{3}{|c|}{ Pericarp thickness (mm) } & \multicolumn{3}{|c|}{ Total soluble solids $\left({ }^{\mathbf{0}} \mathbf{B}\right)$} & \multicolumn{3}{|c|}{ Lycopene (mg/100g) } \\
\hline & & Mean $(\mu)$ & $\left(\mathbf{b}_{\mathbf{i}}\right)$ & $\mathbf{S}^{2} \mathbf{d}_{\mathrm{i}}$ & $\operatorname{Mean}(\mu)$ & $\left(\mathbf{b}_{\mathbf{i}}\right)$ & $\mathbf{S}^{2} \mathbf{d}_{\mathrm{i}}$ & Mean $(\mu)$ & $\left(\mathbf{b}_{\mathbf{i}}\right)$ & $\mathbf{S}^{2} \mathbf{d}_{\mathrm{i}}$ & Mean $(\mu)$ & $\left(\mathbf{b}_{\mathbf{i}}\right)$ & $\mathbf{S}^{2} \mathbf{d}_{\mathrm{i}}$ \\
\hline 1 & Anand & 2.71 & 2.47 & 0.02 & 6.86 & $3.07 *$ & $0.08^{* * *}$ & 4.31 & 0.44 & $0.02 * *$ & 1.79 & 0.66 & $0.04 * *$ \\
\hline 2 & Lehar & 2.64 & $-2.27 * *$ & -0.00 & 6.09 & 3.61 & $0.85 * *$ & 4.02 & $-1.54 * *$ & $0.02 * *$ & 3.02 & 8.66 & $0.65 * *$ \\
\hline 3 & Heem Sohna & 2.51 & -0.16 & 0.01 & 6.57 & 1.40 & $0.05 *$ & 3.94 & 1.76 & $0.03^{* *}$ & 3.63 & -5.90 & $4.84 * *$ \\
\hline 4 & Karan & 2.86 & 3.06 & $0.20 * *$ & 6.35 & 1.03 & -0.00 & 3.52 & 2.92 & $0.17 * *$ & 2.45 & $6.20 * *$ & $0.07 * *$ \\
\hline 5 & Tokita & 3.89 & $-3.30 * *$ & $0.03 *$ & 5.47 & $0.38 *$ & -0.01 & 3.36 & 0.82 & $0.09^{* *}$ & 2.42 & -3.87 & $1.34 * *$ \\
\hline 6 & Rupali & 3.27 & 0.80 & 0.01 & 6.20 & 0.52 & $0.80 * *$ & 4.08 & 2.39 & $0.09^{* * *}$ & 2.31 & -3.22 & $0.67 * *$ \\
\hline 7 & Sonali & 2.84 & 2.59 & $0.05 * *$ & 5.91 & 2.97 & $0.39 * *$ & 3.69 & 1.05 & $0.09 * *$ & 2.50 & 3.66 & $0.41 * *$ \\
\hline 8 & Maharishi & 2.39 & 0.52 & $0.02 *$ & 5.61 & $3.09 *$ & -0.03 & 4.17 & 1.74 & $0.05^{* * *}$ & 2.61 & -4.44 & $0.83 * *$ \\
\hline 9 & Aditya & 2.29 & 0.03 & 0.02 & 6.59 & 0.63 & $0.54 * *$ & 3.92 & 0.09 & $0.07 * *$ & 5.22 & -0.38 & $3.24 * *$ \\
\hline 10 & Kubergeeta & 2.97 & 2.06 & 0.01 & 6.75 & 4.15 & $1.29 * *$ & 3.84 & 2.46 & $0.75^{* * *}$ & 3.13 & 0.23 & $0.05 * *$ \\
\hline 11 & NS- 2535 & 2.46 & $0.14 * *$ & -0.01 & 6.73 & 0.85 & $1.13 * *$ & 4.19 & 1.24 & $0.54 * *$ & 2.98 & 4.96 & $4.69 * *$ \\
\hline 12 & US- 3383 & 3.04 & 2.27 & $0.11 * *$ & 5.56 & -1.23 & $0.36^{* *}$ & 3.74 & 2.21 & $0.18^{* *}$ & 3.42 & -2.78 & $0.62 * *$ \\
\hline 13 & DVRT-2 & 3.65 & 6.92 & $0.66 * *$ & 4.92 & -1.01 & $1.19^{* *}$ & 4.53 & 2.32 & $0.16^{* *}$ & 2.65 & 6.54 & $2.27 * *$ \\
\hline 14 & Solan Lalima & 2.56 & -0.58 & $0.07 * *$ & 6.39 & $-1.40 *$ & $0.04 *$ & 4.42 & 0.96 & $0.11^{* *}$ & 3.64 & 1.70 & $0.44 * *$ \\
\hline 15 & Arka Abha & 4.23 & 0.27 & $0.48 * *$ & 5.54 & 3.42 & $0.20 * *$ & 4.24 & 0.58 & $0.25^{* * *}$ & 2.88 & $-4.98^{*}$ & $0.19 * *$ \\
\hline 16 & Leh Local & 2.42 & 0.58 & -0.01 & 6.56 & 0.91 & $0.05^{*}$ & 4.34 & 2.01 & $0.10^{* *}$ & 3.85 & 10.26 & $1.32 * *$ \\
\hline 17 & Angoorlata & 2.41 & 1.75 & $0.14 * *$ & 4.62 & -0.22 & $0.26 * *$ & 4.37 & 0.21 & $0.29 * *$ & 3.59 & 1.30 & $1.25 * *$ \\
\hline 18 & Arka Saurabh & 3.44 & 0.78 & $0.13 * *$ & 5.19 & 2.88 & $0.69 * *$ & 4.12 & -0.71 & $0.11 * *$ & 2.67 & 1.55 & $0.86 * *$ \\
\hline 19 & Pusa Ruby & 3.42 & 0.93 & $0.02 *$ & 4.13 & 3.80 & $0.81 * *$ & 4.70 & $-1.92 *$ & $0.21 * *$ & 3.03 & 2.49 & $0.41 * *$ \\
\hline 20 & Marglobe & 4.23 & 1.64 & $0.07 * *$ & 4.20 & 1.20 & $0.18^{* * *}$ & 4.03 & 1.04 & $0.16^{* *}$ & 2.73 & 0.22 & 0.00 \\
\hline 21 & Arka Vikas & 4.42 & -0.03 & $0.50 * *$ & 4.98 & 2.11 & $0.84 * *$ & 5.02 & 0.35 & $0.44 * *$ & 2.50 & 0.28 & $0.40 * *$ \\
\hline 22 & Swarna Lalima & 3.73 & $-2.28 * *$ & $0.03 *$ & 4.82 & 1.01 & $0.11 * *$ & 4.04 & -0.68 & $0.21^{* * *}$ & 1.36 & -0.66 & $0.04 * *$ \\
\hline 23 & \begin{tabular}{|l|} 
Swarna \\
Naveen \\
\end{tabular} & 3.88 & 4.83 & $0.45 * *$ & 4.78 & $-4.15^{*}$ & $0.50 * *$ & 4.15 & 2.29 & $0.54 * *$ & 2.21 & -0.28 & $0.01 * *$ \\
\hline 24 & Arka Meghali & 3.25 & -1.37 & $0.74 * *$ & 3.85 & $-1.41^{*}$ & $0.09 * *$ & 4.19 & 2.32 & $0.37 * *$ & 2.46 & 3.35 & $2.10 * *$ \\
\hline 25 & Naveen & 3.34 & $3.32 *$ & $0.02 *$ & 5.08 & -2.64 & $1.08^{* *}$ & 4.39 & 0.56 & $0.29 * *$ & 4.56 & -0.55 & $0.04 * *$ \\
\hline & Mean & 3.15 & 1.00 & & 5.59 & 1.00 & & 4.13 & 1.00 & & 2.94 & 1.00 & \\
\hline
\end{tabular}

*significant at $5 \%$ level of significance, ** significant at $1 \%$ level of significance 
Table.4 Mean value, regression coefficient (bi) and variation due to deviation (s2di) for 25 tomato genotypes

\begin{tabular}{|c|c|c|c|c|c|c|c|c|c|c|}
\hline & & \multicolumn{3}{|c|}{ Beta carotene $(\mathrm{mg} / 100 \mathrm{~g})$} & \multicolumn{3}{|c|}{ Ascorbic acid (mg/100g) } & \multicolumn{3}{|c|}{$\mathbf{p H}$} \\
\hline S. No. & Genotype & Mean $(\mu)$ & $\left(\mathbf{b}_{\mathbf{i}}\right)$ & $\mathbf{S}^{2} \mathbf{d}_{\mathrm{i}}$ & Mean $(\mu)$ & $\left(\mathbf{b}_{\mathbf{i}}\right)$ & $\mathbf{S}^{2} \mathbf{d}_{\mathrm{i}}$ & Mean $(\mu)$ & $\left(\mathbf{b}_{\mathbf{i}}\right)$ & $\mathbf{S}^{2} \mathbf{d}_{\mathbf{i}}$ \\
\hline 1 & Anand & 6.48 & 3.15 & $7.05^{* *}$ & 12.59 & 0.88 & $4.33 * *$ & 4.22 & 1.70 & $0.01 * *$ \\
\hline 2 & Lehar & 4.14 & -1.93 & $8.20 * *$ & 20.41 & -2.22 & $10.28 * *$ & 4.48 & $1.62 * *$ & $-0.00 * *$ \\
\hline 3 & Heem Sohna & 5.44 & 2.65 & $5.12 * *$ & 17.71 & 1.35 & $0.84 * *$ & 4.18 & 1.30 & 0.00 \\
\hline 4 & Karan & 4.47 & $6.92 * *$ & $1.01 * *$ & 15.43 & $-0.07 *$ & 0.16 & 4.21 & 0.01 & $0.00 * *$ \\
\hline 5 & Tokita & 4.54 & 1.60 & $7.43 * *$ & 20.72 & 0.28 & $1.87 * *$ & 4.20 & $1.68 *$ & 0.00 \\
\hline 6 & Rupali & 3.31 & 3.23 & $0.70 * *$ & 16.85 & 0.11 & $0.31 *$ & 4.35 & 1.78 & $0.00 *$ \\
\hline 7 & Sonali & 5.23 & -1.12 & $2.02 * *$ & 10.96 & -0.08 & $0.66 * *$ & 4.21 & $0.26 * *$ & -0.00 \\
\hline 8 & Maharishi & 1.59 & 0.31 & 0.05 & 22.97 & -0.38 & $7.70 * *$ & 4.09 & $0.10 * *$ & -0.00 \\
\hline 9 & Aditya & 6.40 & 4.47 & $4.56^{* * *}$ & 18.20 & 3.24 & $5.39 * *$ & 4.12 & $-0.80 * *$ & $0.00 *$ \\
\hline 10 & Kubergeeta & 1.75 & 0.29 & $0.07 *$ & 15.37 & 1.47 & $18.53 * *$ & 4.43 & 1.01 & 0.00 \\
\hline 11 & NS- 2535 & 5.14 & 4.28 & $7.79 * *$ & 17.09 & $-0.34 *$ & $0.40 * *$ & 4.41 & 1.03 & 0.00 ** \\
\hline 12 & US- 3383 & 6.40 & 3.86 & $5.55 * *$ & 15.33 & -0.11 & $0.44 * *$ & 4.34 & $2.20 * *$ & 0.00 \\
\hline 13 & DVRT-2 & 1.77 & 0.72 & 0.04 & 22.73 & 4.30 & $4.74 * *$ & 4.32 & 1.39 & 0.00 \\
\hline 14 & Solan Lalima & 7.06 & $0.04 * *$ & -0.00 & 20.31 & 2.24 & $0.79 * *$ & 4.10 & 0.92 & 0.00 \\
\hline 15 & Arka Abha & 4.19 & $4.74 * *$ & $0.14 * *$ & 25.51 & 1.21 & $1.61 * *$ & 4.25 & 1.26 & 0.00 \\
\hline 16 & Leh Local & 4.95 & 5.13 & $3.33 * *$ & 26.07 & 0.31 & $1.43 * *$ & 4.37 & 1.32 & -0.00 \\
\hline 17 & Angoorlata & 3.45 & -4.44 & $6.15^{* *}$ & 25.03 & $5.85 * *$ & $1.05 * *$ & 4.29 & 0.48 & 0.00 \\
\hline 18 & Arka Saurabh & 6.06 & $-1.77 *$ & $1.09 * *$ & 23.71 & -0.63 & $2.42 * *$ & 4.32 & 1.04 & 0.00 \\
\hline 19 & Pusa Ruby & 2.56 & -3.85 & $6.87 * *$ & 19.04 & $4.41 *$ & $3.27 * *$ & 4.07 & 0.56 & 0.00 \\
\hline 20 & Marglobe & 6.30 & 0.02 & $5.27 * *$ & 20.11 & 2.24 & $15.80^{* *}$ & 4.41 & $1.76^{*}$ & 0.00 \\
\hline 21 & Arka Vikas & 7.60 & -1.67 & $1.13 * *$ & 22.82 & 1.34 & $3.51 * *$ & 4.49 & 1.88 & $0.00 * *$ \\
\hline 22 & Swarna Lalima & 6.64 & 1.49 & $1.29 * *$ & 27.17 & -0.65 & $4.32 * *$ & 4.28 & 0.26 & $0.00 * *$ \\
\hline 23 & Swarna Naveen & 5.10 & -1.37 & $1.81 * *$ & 23.63 & -0.34 & $0.76 * *$ & 4.09 & 1.03 & 0.00 ** \\
\hline 24 & Arka Meghali & 4.34 & -2.52 & $6.26 * *$ & 27.96 & 0.31 & $0.43 * *$ & 4.45 & 1.03 & 0.00 \\
\hline 25 & Naveen & 2.58 & 0.74 & $0.53 * *$ & 15.22 & 0.26 & $0.32 *$ & 4.13 & $0.06^{*}$ & $0.00 *$ \\
\hline & Mean & 4.70 & 1.00 & & 20.12 & 1.00 & & 4.27 & 1.00 & \\
\hline
\end{tabular}

*significant at $5 \%$ level of significance, $* *$ significant at $1 \%$ level of significance 
Whereas rest of the genotypes showed significant deviation from regression line which revealed significance of unpredictable components.

For betacarotene content $(\mathrm{mg} / 100 \mathrm{~g})$ genotype Solan Lalima had regression coefficient lesser than one which showed its preference to unfavourable environments. Genotype Arka Vikas had the highest betacarotene (7.60 $\mathrm{mg} / 100 \mathrm{~g}$ ) content than the average mean $(4.70 \mathrm{mg} / 100 \mathrm{~g})$ and Maharishi recorded the lowest betacarotene content $(1.59 \mathrm{mg} / 100 \mathrm{~g})$. Among hybrids it varied from 6.48 to $1.59 \mathrm{mg} / 100 \mathrm{~g}$ and 7.60 to $1.77 \mathrm{mg} / 100 \mathrm{~g}$ in open pollinated genotypes. Cheema et al., (2013) revealed betacarotene in the range of $4.12-3.57 \mathrm{mg} / 100 \mathrm{~g}$ in 26 hybrids under open field conditions. Dar and Sharma (2011) reported a range of $1.08-2.55 \mathrm{mg} / 100 \mathrm{~g}$ in 60 genotypes under study.

As regards to ascorbic acid content (mg/100g) genotype Karan with regression coefficient lesser than one, non significant deviation from regression line which is suitable for growing under unfavouarble environment but had lesser mean value. Rest of the genotypes showed significant component of non linear response of $\mathrm{G} \times \mathrm{E}$ interaction. Genotype Arka Meghali had maximum ascorbic acid (27.96 $\mathrm{mg} / 100 \mathrm{~g}$ ) content as compared to average mean $(20.11 \mathrm{mg} / 100 \mathrm{~g})$. While the minimum was noticed in Sonali $(10.96 \mathrm{mg} / 100 \mathrm{~g})$. Among hybrids it ranged from 22.97 to $10.96 \mathrm{mg} / 100 \mathrm{~g}$ and 27.96 to $19.04 \mathrm{mg} / 100 \mathrm{~g}$ in open pollinated genotypes. Cheema et al.,(2013) revealed ascorbic acid in the range of $20.62-12.50 \mathrm{mg} / 100 \mathrm{~g}$ in 26 hybrids under study. Zahedi et al., (2012) recorded a range of $23.57-31.62 \mathrm{mg} / 100 \mathrm{~g}$ in ten genotypes; Hazarika and Phookan (2009) obtained ascorbic acid content from 6.61-16.56 mg/100; Revanasiddappa (2008) found 19.09 $\mathrm{mg} / 100 \mathrm{~g}$.

Genotypes Kubergeeta, Arka Saurabh and
Arka Meghali had regression coefficient close to one with non significant deviation from regression line which were widely adapted to all the environments under study for fruit $\mathrm{PH}$. Genotypes Heem Sohna, DVRT-2, Arka Abha, Leh Local and Marglobe showed regression coefficient greater than unity with non significant deviation from regression line and are suitable for favourable environments. Genotypes Solan Lalima, Angoorlata and Pusa Ruby had regression coefficient lesser than unity which are suitable for recommendation to unfavourable environments. Based on mean value, genotype Arka Vikas (4.49) recorded maximum $\mathrm{pH}$ than average mean (4.27) and lowest $\mathrm{pH}$ was noticed in Pusa Ruby (4.07). Among hybrids it ranged from 4.48 to 4.09 and 4.49 to 4.07 for open pollinated genotypes. Jyothi et al., (2012) found $\mathrm{pH}$ varying from 3.35-3.65; Mane et al., (2010) observed $\mathrm{pH}$ (4.30); Ashwini (2005) reported $\mathrm{pH}$ content for parents ranged from 3.21 to 4.34 and for hybrids from 3.04 to 4.89 .

Considering the overall performance for quality traits, genotype Rupali was identified as widely adapted to all environments for number of locules per plant; genotypes Karan for pericarp thickness and genotypes Kubergeeta, Arka Saurabh and Arka Meghali were identified as stable for fruit $\mathrm{pH}$. Based on mean value,genotype Aditya had highest lycopene content; genotype Arka Vikas had highest betacarotene content and genotpype Arka Meghali had highest ascorbic acid content depicting its suitability for processing purpose.

\section{References}

Adedeji, O., Taiwo, K.A., Akanbi, C.T. and Ajani, R. 2006. Physicochemical properties of four tomato cultivars grown in Nigeria. J. Food Processing and Preservation, 30(1): 79-86. 
Akinfasoye, J., Dotun, A., Ogunniyan, J., and Ajayi, E.O. 2011. Phenotypic relationship among agronomic characters of commercial tomato (Lycopersicum esculentum) hybrids. American-Eurasian J. Agronomy, 4(1): 17-22.

Aravindakumar, J.S., Mulge, R., Madalageri, H.B., Patil, M.P., Kulkarni, M.S. and Gangadharappa, P.M.2001. Stability differences among tomato genotypes for fruit quality parameters, Vegetable Sci., 28: 102-105.

Ashwini, M. C. 2005. Heterosis and combining ability studies for heat tolerance in tomato. M.Sc. (Agri.) Thesis. University of Agriculture Science, Dharwad (India).

Bai, Y., and Lindhot, P. 2007. Domestication and breeding of tomatoes: what have we gained and what can we gain in the future. Annals of Botany, 100(5): 10851094.

Cantore, V., Boari, F., Vanadia, S., Pace, B., Palma, E-de, Leo, L. and Zacheo, G. 2008. Evaluation of yield and qualitative parameters of high lycopene tomato cultivars. Acta Horticulturae, 789: 173-179.

Cheema, D. S., Singh, N. and Jindal, S. K. 2013. Evaluation of indeterminate tomato hybrids for fruit, yield and quality traits under net house and open field conditions. Vegetable Sci., 40(1): 45-49.

Dar, R.A. and Sharma, J.P. 2011. Genetic Variability Studies of Yield and Quality Traits in Tomato (Solanum lycopersicum L.). Int. J. Plant Breeding and Genetics, 5(2): 168-174.

Das, R.M., Hossain, T.M.M., Sultana, M.M., Sarwar, G.S.H.M. and Hafiz, M.H.R. 2011. Effect of different sowing time on the quality of tomato varieties. Bangladesh Res. Publish J., 6(1): 46-51. Dufera, J.T.2013. Evaluation of Agronomic
Performance and Lycopene Variation in Tomato (Lycopersicon esculantum Mill.) Genotypes in Mizan, Southwestern Ethiopia. World Appl. Sci. J., 27(11).

Eberhart, S. A. and Russell, W. A. 1966. Stability parameters for comparing varieties. Crop sci., 6: 36-40.

George, B., Kaur, C., Khurdiya, D. S. Kapoor, H. C. 2004.Antioxidants in tomato (Lycopersicon esculentum Mill) as a function of genotype. Food Chemistry, 84:45-51.

Giovannucci, E., Erdman, W., Schwartz, J., Clinton, K. and Miller, E.2002. Tomato products, lycopene and prostate cancer risk. Urologic clinics North America, 29(1): 83-93.

Hazarika, T.K. and Phookan, D.B. (2009). Adaptability of different tomato cultivars during off-season under protected cultivation. Vegetable Sci., 36(3): 386-389.

Jyothi, H.K., Patil, M.G. and Santhosha, H.M. 2012. Studies on stability of processingtype genotypes of tomato (Solanum lycopersicum L.). J. Horticulture Sci., 7(2):138-141.

Kalloo, G., Chaurasia., S.N.S. and Singh, M.1998. Stability analysis in tomato. Vegetable Sci., 25(1):81-84.

Kumari, S. and Sharma, M.K. 2011. Exploitation of heterosis for yield and its contributing traits in tomato (Solanum lycopersicum L.). Int. J. Farm Sci., 1(2): 45-50.

Lutsenko, E.A., Carcamo, J.M., Golde,D.W. 2002.Vitamin C prevents DNA mutation induced by oxidative stress. $J$. Biol. Chem., 277:16895-16899.

Maitidevi, M., and Kathmmandu, M. 2008. Product chain study tomato (p. 480). Ministry of Agriculture and Cooperatives, Project Management Unit, Biratnagar, Nepal.

Mane. R., Sridevi,O., Salimath, P.M., 
Deshpande, S.K. and Khot, A.B. 2010. Performance and stability of different tomato (Solanum lycopersicum) genotypes. Indian J. Agri. Sc., 80(10): 898-901.

Marchioli, R., Schweiger, C., Levantesi, G., Tavazzi, L., Valagussa, F. 2001. Antioxidant vitamins and prevention of cardiovascular disease: epidemiological and clinical trial data. Lipids, 36: S53S63.

Miller, E.C., Hadley, C.W., Schwartz, S.J., Erdman, J.W., Boileau, T.M.W. and Clinton, S.K.2002.Lycopene, tomato products, prostrate cancer prevention. Have we established causality? Pure and Appl. Chem., 74(8): 1435-1441

Nwosu, D. J., Onakoya, O. A., Okere, A. U., Babatunde, A. O. and Popoola, A. F. 2014. Genetic variability and correlations in rainfed tomato (Solanum spp.) accessions in Ibadan, Nigeria. Greener J. Agricultural Sci., 4(5): 211219.

Panthee, D. R., Cao, C. X., Debenport, S. J., Rodríguez, G. R., Labate, J. A., Robertson, L. D., Breksa, A. P., III, Knaap, E. van der, Gardener, B. B. M. O. 2012. Magnitude of genotype $\times$ environment interactions affecting tomato fruit quality, Hort. Sci., 47(6): 721-726.

Prasanna, H.C., Chaubey, T., Kumar, R., Rai,
M., Verma, A. and Singh, S. 2007. Identification of stable variety for yield and quality attributes in tomato. Vegetable Sci., 34: 131-134.

Ranganna, S. 1976.Manual of Analysis of Fruits and Vegetables products, Tata McGraw Hill Co. Pvt. Ltd., New Delhi.p.94-98.

Revanasiddappa, K.V. 2008. Breeding investigations involving biparental mating and selection approaches in tomato (Solanum lycopersicum Mill.). M.Sc. (Agri.) Thesis. University of Agriculture Science, Dharwad

Rick, C. M. 1969. Origin of cultivated tomato, current status of the problem. Abstract XI Int. Botanical Congress p. 180.

Sadasivam, S. and Manickam. 1992. Biochemical methods ( $2^{\text {nd }}$ edition). New age international (p) limited.

Thapliyal, A., Singh, J. P. 2009. Stability analysis for growth, yield and quality characters of tomato (Solanum lycopersicum L). Pantnagar $J$ Res., 7(2): 180-183.

Zahedi, S.M., Ansari, N.A. and Eftekhari, S.A. 2012. Investigation of yield and adaptation of ten selected genotypes of tomato under subtropical climate conditions (Ahvaz). J. Food, Agri. Environ., 10(1): 782-786.

\section{How to cite this article:}

Sonam Spaldon, R.K. Samnotra, Rinchan Dolkar and Deshraj Choudhary. 2017. Stability Analysis and Genotype X Environment Interaction of Quality Traits in Tomato (Solanum lycopersicum L.). Int.J.Curr.Microbiol.App.Sci. 6(2): 1506-1515. doi: http://dx.doi.org/10.20546/ijcmas.2017.602.168 